\title{
Clampless laparoscopic partial nephrectomy: a step towards a harmless nephron-sparing surgery?
}

\author{
Francesco Porpiglia, Riccardo Bertolo, Ivano Morra and Cristian Fiori \\ Division of Urology - "San Luigi Gonzaga” Hospital - Orbassano (Turin), Italy
}

\section{ABSTRACT}

Purpose: To evaluate the results of our technique of clampless laparoscopic partial nephrectomy (LPN) and its impact as an emerging treatment for small renal masses (SMRs). Materials and Methods: We reviewed our prospectively maintained database: data of 117 patients who consecutively underwent LPN at our Institution from January 2009 to December 2011 were studied. Patients were divided into 2 Groups based on operative technique: Group A: clampless-LPN (cl-LPN); Group B: conventional LPN (clamping of renal artery). Demographic and peri-operative data, complications, pre- and post-operative serum creatinine and estimated glomerular filtration rate (eGFR) were registered and compared by Student's t- and Chi-square-tests (p-values $<0.05$ considered statistically significant).

Results: 41 patients were in Group A and 76 in Group B. Groups were comparable in terms of preoperative data except for tumour's size ( $2.35 \pm 1.10$ vs. $3.19 \pm 1.57$, Group A vs. B, respectively, $p=0.0029$ ). Concerning perioperative data, warm ischemia time (WIT) was 0 min. in all Group A cases; mean WIT in Group B was $20.90 \pm 9.27 \mathrm{~min}$. One case (2.4\%) in Group A (central tumour) was converted to conventional LPN. Mean eGFR postoperative decrease was higher in Group B $(0.17 \pm 9.30$ vs. $4.38 \pm 11.37$ mL/min., A vs B, respectively, $\mathrm{p}=0.0445)$.

Conclusions: Notwithstanding the limits of the study, our results suggest that cl-LPN is a safe and effective technique, which allows surgeon to surgically treat SRMs even in case of complex location, without injuring kidney by ischemia.

\section{ARTICLE INFO}

\section{Key words:}

Laparoscopy;

Nephrectomy; Ischemia

Int Braz J Urol. 2012; 38: 480-8

Submitted for publication:

March 28, 2012

Accepted after revision:

July 23, 2012

\section{INTRODUCTION}

It has been proved that partial nephrectomy (PN) is the treatment of choice in cases of surgical, clinical T1 tumors $(<7 \mathrm{~cm})$ (1). During PN, whole renal hilum or renal artery alone clamping is typically performed, because it is vital to decrease blood losses, allowing improved visualization, which is paramount to achieve negative oncological margins and to perform precise kidney reconstruction.
On the other side, such clamping causes renal ischemia that could lead to some degree of renal ischemic damage. The critic threshold for the beginning of such damage has been extensively studied and fixed around 20-25 minutes (2). Nevertheless, recent studies demonstrated that every minute is crucial in determining renal damage during renal ischemia (3) and stimulated surgeons towards the introduction of new technical tricks in order to further reduce warm ischemia time (WIT) $(4,5)$ and lead to the conception of the development of LPN performed with "zero-ischemia"- 
technique, with the aim of completely eliminate surgical renal ischemia.

Some authors firstly described the technique of clampless LPN (cl-LPN) in 2003 (6,7), but a modified one has been recently published incorporating selective branch micro-dissection of renal artery/vein, coupled with carefully calibrated and timed intraoperative reduction of blood pressure (8).

In the present study, we retrospectively evaluated our experience with cl-LPN: the primary aim was to evaluate the results of this technique; the secondary aim was to compare results of cl-LPN to conventional LPN ones.

\section{MATERIAL AND METHODS}

We retrospectively reviewed our prospectively-maintained, institutional review boardapproved database of LPN procedures performed since January 2001 and we extracted data from January 2009 till December 2011. During this period all PNs were performed by using laparoscopic technique in our Institution.

\section{Indications for cl-LPN}

Till September 2011 cl-LPN was performed only in case of lesion with exophytic growth (defined $\geq 50 \%$ exophytic, score 1 according to PADUA score (9)) clinically staged T1a. On the contrary, from September 2011 to the end of the studied period we extended the indication to all SRMs except by central lesions completely surrounded by healthy renal parenchyma (defined entirely endophytic, score 3 according to PADUA score).

Patients were divided into two groups according to operative technique: Group A included patients who underwent clampless technique (clLPN); Group B included patients treated by conventional technique (with clamping of the renal artery).

\section{Surgical Technique}

Conventional technique has been previously described (10).

Differences when performing the clampless technique were as follows: neither renal hilum nor renal artery alone were clamped but they were accurately dissected till their initial branches; carbon dioxide pressure was raised till $20 \mathrm{mmHg}$ during lesion's excision and tumour was slowly resected by cold scissors; dissection of the tumour along the pseudocapsule was performed by forceps and with the help of suction device (Elefant, Coloplast GmBh, Hamburg Germany). During the resection, vessels emerging from resection bed were coagulated with bi-polar forceps (Storz Medical System, Tuttlingen, Germany) or clipped by Hem-o-lock clips (Weck Closure Systems, Research Triangle Park, NC, USA). No dedicated anaesthetic procedures (such as calibrated hypotension) were used neither during cl-LPN nor during conventional LPN.

All procedures were performed by the same surgeon (F.P.).

An year-by-year evaluation of the absolute number and rate of cl-LPN with respect to conventional LPN performed by warm ischemia (WI) was done. For each patient, we registered demographic data and co-morbidities classified according to Charlson's co-morbidity index (CCI) (11)). Radiological tumour size (according to Computed Tomography scan, CT-scan), side and location were considered and lesions were classified according to RENAL nephrometry score (12) and PADUA score (9). Peri-operative, histopathological data and post-operative complications (classified according to Clavien's System (13)) were also recorded. Finally, serum creatinine ( $\mathrm{SCr}$ ) was recorded preoperatively and at hospital discharge. Estimated glomerular filtration rate (eGFR) was calculated by using the modification of diet in renal disease (MDRD) formula (14). These variables were used as surrogates of renal function.

Means and standard deviations were used in order to summarise continuous variables; frequencies and proportions were used in order to summarise categorical variables. Student's ttest was used to test the differences between the means of continuous variables after verifying that analysed variables were approximately normally distributed; the differences between frequencies and proportions were tested using Chi-square test. All tests were two-sided, and p-values $<0.05$ were considered significant. "Statistica" software program Ver. 6.0 (Tulsa, OK, USA) was used to perform the whole statistical analysis. 


\section{RESULTS}

One hundred and seventeen procedures of LPN were performed in our Institution during the revised period.

Among the 117 patients who underwent LPN, 41 underwent cl-LPN and constituted Group A. The remaining 76 patients underwent conventional LPN with clamping of renal artery and composed Group B. In our case-study, it was observed a trend towards an increasing choice in favour of cl-LPN along the revised period (Figure-1).

Concerning Group A, CT-scan lesion size was $2.35 \pm 1.10 \mathrm{~cm}$, operative time $133.53 \pm 40.54$ min. and estimated blood losses (EBL) $201.46 \pm$ $109.81 \mathrm{~mL}$. One case $(2.4 \%)$ of central angiomyolipoma was converted to conventional LPN due to complex bleeding requiring clamping of renal artery: this event was recorded as an intraoperative complication. We recorded only two medical $(4.8 \%)$ post-operative complications ( 1 fever and 1 bronchitis); none of them was $\geq$ III Clavien grade (13). In four patients (9.7\%) the reconstruction of parenchymal defect at the end of tumour excision was not performed. Complete results of cl-LPN are reported in Table- 1.

Results of this technique were then compared with the results of conventional LPN
(Table-1). Groups were comparable except for tumour's size that was significantly higher in Group B $(2.35 \pm 1.10$ vs. $3.19 \pm 1.57$, Group A vs. $\mathrm{B}$, respectively, $\mathrm{p}=0029)$. On the contrary, no differences were recorded either in terms of RENAL nephrometry or PADUA scores. WIT was $20.90 \pm 9.27 \mathrm{~min}$. in Group B and was equal to 0 in all Group A cases ( $p<0.0001)$. Peri-operative variables were comparable. Particularly, operative time $(133.53 \pm 40.54$ vs $120.59 \pm 32.33$, A vs. B, respectively) and EBL (201.46 \pm 109.81 vs. $164.27 \pm 128.68$, A vs. B, respectively) were slightly higher in Group A even if these differences did not reach statistical significance $(\mathrm{p}=$ 0.0763 and $p=0.1464$, respectively). No differences were noted between the two Groups in terms of post-operative complications. Similarly, the rate of positive surgical margins (PSMs), thickness of peri-tumoral healthy tissue excised and distribution of histotypes at histopathological assessment (Figure-2) were not statistically different. Finally, concerning functional results, we did not find any differences when comparing pre-operative and post-operative $\mathrm{SCr}$ and eGFR in both Groups, even if a trend towards a more relevant post-operative worsening of eGFR was observed in Group B with respect to Group A ( $p=$ 0.0445 , Figure-3). Complete functional results are reported in Table-2.

Figure 1 - Histogram showing the annual rate of choice of clampless laparoscopic partial nephrectomy (LPN) with respect to the overall annual number of LPN procedures performed (annual absolute number of procedures is reported in brackets). In Red color: clampless LPN; in Blue color: conventional LPN.

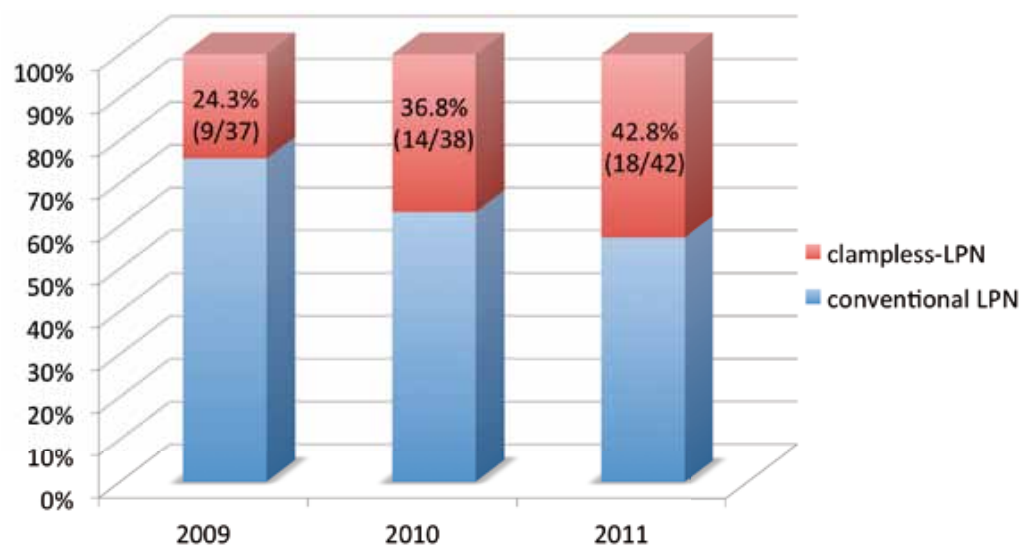


Table 1 - Demographic, peri-operative, post-operative variables and complications.

\begin{tabular}{|c|c|c|c|}
\hline & $\begin{array}{c}\text { Group A } \\
(n=41)\end{array}$ & $\begin{array}{c}\text { Group B } \\
(n=76)\end{array}$ & $\mathrm{p}$-value \\
\hline Age, mean (SD), years & $65.09(7.47)$ & $61.26(13.68)$ & 0.1371 \\
\hline Males, no. (\%) & $26(63.4)$ & $60(78.9)$ & 0.0642 \\
\hline BMI, mean (SD) & $27.14(3.62)$ & $26.47(3.29)$ & 0.2792 \\
\hline CCI, mean (SD) & $0.91(1.22)$ & $0.93(1.53)$ & 0.9409 \\
\hline ASA score $\geq 3$, no. (\%) & $20(48.8)$ & $40(52.6)$ & 0.6803 \\
\hline CT-scan size, mean (SD), cm & $2.35(1.10)$ & $3.19(1.57)$ & 0.0029 \\
\hline RENAL score, mean (SD) & $5.65(1.65)$ & $5.84(1.86)$ & 0.5790 \\
\hline PADUA score, mean (SD) & $7.09(1.46)$ & $7.48(1.79)$ & 0.2519 \\
\hline Right sided, no. (\%) & $21(51.2)$ & $38(50.0)$ & 0.9180 \\
\hline Retroperitoneal approach, no (\%) & $31(75.6)$ & $61(80.3)$ & 0.6157 \\
\hline Operative time, mean (SD), min. & $133.53(40.54)$ & $120.59(32.33)$ & 0.0763 \\
\hline WIT, mean (SD), min. & 0 & $20.90(9.27)$ & $<0.0001$ \\
\hline $\mathrm{EBL}$, mean (SD), $\mathrm{mL}$ & $201.46(109.81)$ & $164.27(128.68)$ & 0.1464 \\
\hline Intra-op.ve complications, no. (\%) & $1(2.4)$ & $1(1.3)$ & 0.6556 \\
\hline Post-op.ve complications, no. (\%) & $2(4.8)$ & $11(14.5)$ & 0.1380 \\
\hline Post-op.ve complications $\geq$ III Clavien's grade, no. (\%) & $0(0)$ & $0(0)$ & -- \\
\hline A.P. size, mean (SD), cm & $2.43(1.13)$ & $3.45(1.65)$ & 0.0006 \\
\hline $\begin{array}{l}\text { Thickness of peri-tumoural healthy tissue excised, } \\
\text { mean (SD), mm }\end{array}$ & $2.46(1.39)$ & $3.06(2.94)$ & 0.2365 \\
\hline Malignant lesions, no. (\%) & $27(65.8)$ & $60(78.9)$ & 0.1268 \\
\hline PSMs, no. (\%) & $1(2.4)$ & $2(2.6)$ & 0.7485 \\
\hline
\end{tabular}

BMI: body mass index; CCI: Charlson's index; CT: computed tomography; ASA: American Society of Anesthesiology; WIT: warm ischemia time; EBL: estimated blood loss; A.P: anatomo-pathological; PSMs: positive surgical margins; SD: standard deviation.

\section{DISCUSSION}

Common tendency in urological literature is an insistence towards a reduction in WIT, because WI has been recognised as the most important risk factor in determining a loss of renal function in post-partial setting (15).

For this reason, lots of studies concerning looking for a "safe" cut-off for WIT $(2,3)$ and tech- niques aiming to the reduction in WIT $(4,5)$ have been published.

Functional results of PN have demonstrated that renal damage and subsequent loss of renal function occur even in cases of lower WIT, demonstrating that every minute counts when renal hilum is clamped $(3,16)$.

Based on these findings, recently an innovative technique of LPN has been introduced, 
Figure 2 - Histogram showing histotypes' distribution at histopatological analysis. In Red color: clampless LPN; in Blue color: conventional LPN (CC-RCC: clear-cell renal cell carcinoma; PRCC: papillary cell renal cell carcinoma; CHROM: chromophobe renal cell carcinoma; ONCO: renal oncocytomas; AML: renal angiomyolipomas).

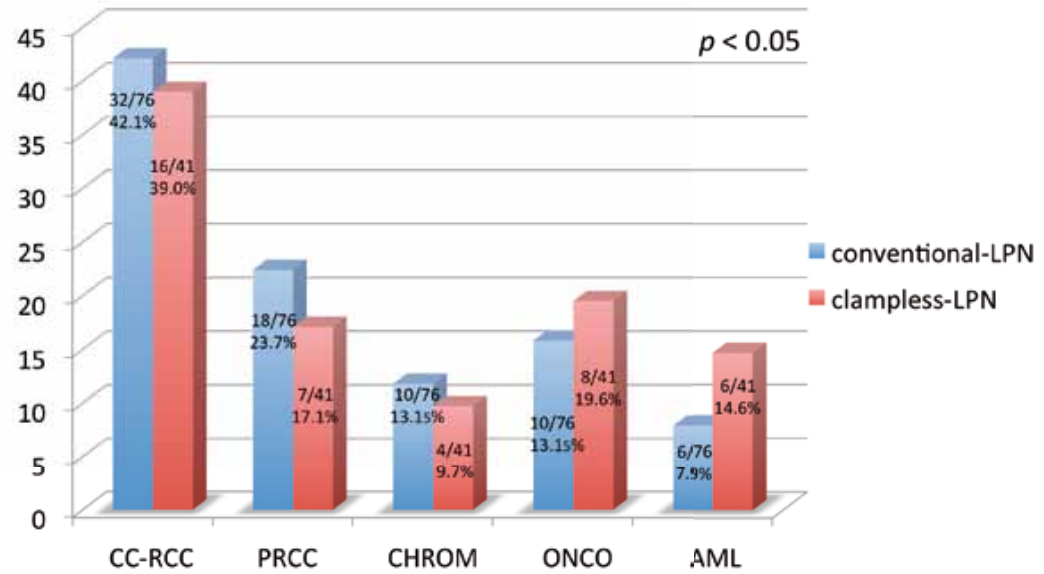

Figure 3 - Plot lines showing the trend between pre-operative and post-operative estimated Glomerular Filtration Rate (eGFR). Red Line: clampless-laparoscopic partial nephrectomy (LPN); Blue Line: conventional LPN.

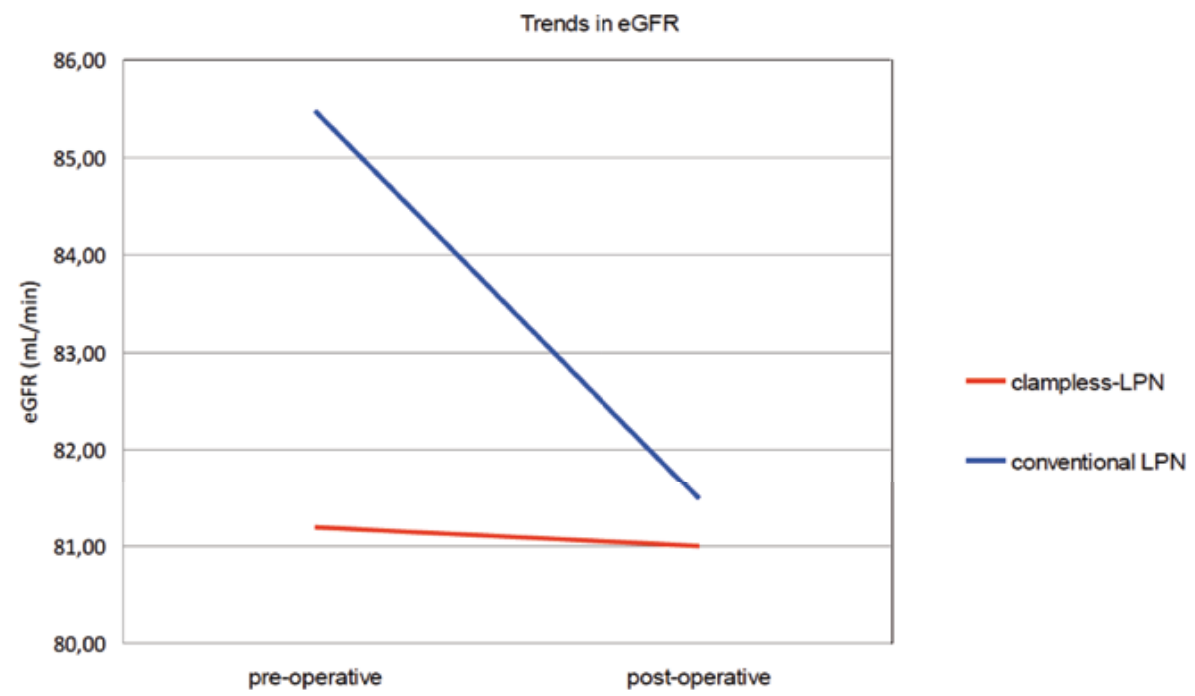

with the purpose of minimally injure the kidney when treating SRMs: Gill et al. described "zeroischemia” PN (8), a nephron-sparing surgical technique based on no-clamping of renal hilum, coupled with calibrated and timed intraoperative reduction of blood pressure. Moreover, Gill et al. described a clip ligation of a tertiary or quaternary renal arterial branch, eventually after micro-nephrotomy (17) and vascular micro-dissection (18). Clipping is achieved by neurosurgical micro-bulldogs on the targeted vessel. This allowed precise identification and isolation of the arterial supply to the mass in question. Even if interesting and described almost three decades ago in various sur- 
Table 2 - Pre-operative and Post-operative functional data.

\begin{tabular}{|c|c|c|c|}
\hline & $\begin{array}{l}\text { Group A } \\
(n=41)\end{array}$ & $\begin{array}{l}\text { Group B } \\
(n=76)\end{array}$ & p-value \\
\hline Pre-op.ve SCr, mean (SD), mg/dL & $0.91(0.20)$ & $0.97(0.24)$ & 0.1750 \\
\hline (p-value t-test pre-op.ve vs post-op.ve values) & 0.8420 & 0.8208 & \\
\hline Post-op.ve SCr, mean (SD), mg/dL & $0.92(0.25)$ & $0.98(0.30)$ & 0.2772 \\
\hline Pre-op.ve eGFR, mean (SD), mL/min. & $81.19(25.71)$ & $85.47(19.37)$ & 0.3128 \\
\hline ( $p$-value t-test pre-op.ve vs post-op.ve values) & 0.9723 & 0.2533 & \\
\hline Post-op.ve eGFR, mean (SD), mL/min. & $81.00(23.64)$ & $81.48(23.34)$ & 0.9160 \\
\hline Decrease in eGFR, mean (SD) $\mathrm{mL} / \mathrm{min}$. & $0.17(9.30)$ & $4.38(11.37)$ & 0.0445 \\
\hline
\end{tabular}

SCr: serum creatinine; eGFR: estimated glomerular filtration rate; SD: standard deviation

gical disciplines (19-22), intraoperative reduction of blood pressure is not always feasible due to the possible vascular comorbidities of patients. Moreover, the use of micro-bulldogs did not completely avoid the kidney from WI and subsequent damage of the healthy tissue.

On the other hand, some authors have already described cl-LPN without intraoperative reduction of blood pressure: in 2003 Guillonneau et al. (6) concluded that LPN could be performed without renal vascular clamping in selected cases; later in 2005 Nadu et al. (7) suggested that clamping of the renal artery should be considered even for very small, superficial tumours. Following these suggestions, in our Institution, since the beginning of our experience with LPN to 2009, a few selected lesions have anecdotally been treated by cl-LPN. Since 2009, we expanded the use of this technique that was introduced in our daily surgical practice with rigorous indications.

The aim of our present study was to describe our technique and results of cl-LPN and to compare its results with those of conventional technique.

We therefore reviewed our prospectively maintained database and we analysed data of 117 consecutive patients treated with LPN by the same surgeon at our Institution. With respect to our conventional technique (10), crucial of clampless technique is: an accurate dissection of hilar renal vessels without vessels clamping, a slower resection of lesion by cold scissors using suction device, allowing immediate suction of any blood loss from resection bed; precise coagulation by bi-polar forceps of any vessel emerging from resection bed during enucleoresection, eventually clipped by Hem-o-lock clips. Rising of carbon dioxide pressure during tumor excision and suture is very helpful during this phase. These tricks altogether allowed us to have an adequate operative field and to avoid parenchymal suture in selected cases.

One important point of our technique is that no dedicated anaesthetic procedure (such as calibrated hypotension) was used during interventions, and this also allows the use of these techniques in patients with vascular comorbidities. Clampless technique has progressively gained a great impact in our case study: it has represented about one-third of surgical choice for the treatment of SRMs since 2010, with the intent in increasing the yearly volume of such intervention in our Centre: in 2011 the trend is towards 50\% of LPN procedures. 
During our statistical analysis, after patients were divided into two Groups according to clamping of the renal artery or not, the groups were substantially comparable except for lesions' size at pre-operative CT-scan. As also confirmed by pathological diameter, lesions excised by cl-LPN were significantly smaller with respect to those excised by conventional LPN. This could be explained by the fact that Group $\mathrm{B}$ is wider and includes extreme challenging cases performed due to absolute indications of the consolidated conventional LPN technique. On the other side, neither RENAL nephrometry score nor PADUA score were different between the Groups, indicating that tumour characteristics considered as a whole were comparable between the Groups, and that even challenging lesions were approached by cl-LPN. Accordingly, Soroush et al. (23) recently demonstrated that clinical stage does not preclude patients from undergoing cl-LPN.

Interestingly, there was a trend toward increase of operative time in Group A. We think that this could be due to a slower tumour resection, which required coagulation and ligation of the resection bed vessels and a slower parenchymal suturing, performed without "anxiety" dictated by ischemia. Mean EBL in Group A were about 200cc and were slightly higher than those in Group B ( $p=0.1464)$. We think that this result is acceptable for this kind of surgery, moreover, regarding the clinical point of view, no blood transfusions were required in Group A and we believe that this is the most important parameter that has to be considered.

One case of significant intra-operative bleeding in our cl-LPN experience occurred, but the accurate dissection of renal hilum and its initial branches performed before the beginning of tumor resection allowed to quickly clamp the renal artery by bulldog clamp and to manage bleeding, converting cl-LPN to conventional LPN.

Another important point was the complication rate: cl-LPN did not show any increase regarding neither intra-operative nor post-operative complications, demonstrating it to be a safe technique. Moreover, no differences were recorded in terms of pathological results, name- ly in terms of PSM, and this is a crucial point when a malignant lesion is managed. We think that these results demonstrate how clampless technique allowed the same "quality" of tumour excision if compared to conventional technique without compromising oncological outcomes.

Finally, concerning functional results, we did not record any differences in terms of $\mathrm{SCr}$ and eGFR when comparing pre-operative with post-operative values in both Groups. Nevertheless, we observed a trend towards a higher eGFR worsening in Group B that was not observed in Group A ( $p=0.0445)$. Even if mean WIT in Group $\mathrm{B}$ was around 20 minutes, a threshold lower than the "safe" one described by urological literature, a loss of renal function estimated by eGFR was detected within the first days postoperatively. This loss was not recorded in patients who underwent clampless technique, confirming that maybe the only way to avoid renal damage following partial nephrectomy is to avoid surgical ischemia. These are preliminary results and we are not able to draw any definitive conclusion, but this is an important feature that can lead to more accurate studies designed on the use of more precise tools to evaluate renal function.

Our study has some limitations because a limited number of patients have been enrolled and Groups were not completely comparable. Moreover, a selection bias has been done in the selection of patients candidate to cl-LPN, most of all first cases treated by this technique. Nevertheless, neither RENAL nor PADUA score were statistically different between the Groups, and this fact softened this bias. Although these limitations could affect our results, we believe that our study provided useful information to the urologic community: cl-LPN is an emerging alternative to conventional LPN that could be a new surgical option when approaching SRMs. By this approach kidney does not undergo WI and this, reasonably, limits renal damage without significant increase of blood losses and complications or impairment of pathological results.

In our opinion, it is essential that the surgeon has significant laparoscopic experience before embarking on this kind of procedure, especially for the most challenging cases. 


\section{CONCLUSIONS}

In our experience, cl-LPN is a safe and effective technique, allowing management of differently located tumours without injuring kidney by ischemia and we suggest it to be considered whenever approaching a SMR.

We recommend a consolidated experience in conventional LPN before embarking in this kind of technique because advanced skills in reconstructive laparoscopic techniques are required to achieve acceptable results. Finally, we think that this technique could represent a significant step toward (virtually) harmless nephron-sparing surgery even if further studies have to be planned in order to confirm this statement.

\section{ABBREVIATIONS}

LPN: Laparoscopic Partial Nephrectomy;

SRMs: Small Renal Masses;

cl-LPN: clampless-Laparoscopic Partial Nephrectomy;

eGFR: estimated Glomerular Filtration Rate;

WIT: Warm Ischemia Time;

PN: Partial Nephrectomy;

WI: Warm Ischemia;

CT: Computed Tomography;

CCI: Charlson's Comorbidity Index;

SCr: Serum Creatinine;

MDRD: Modification of Diet in Renal Disease;

EBL: Estimated Blood Losses;

PSMs: Positive Surgical Margins.

\section{CONFLICT OF INTEREST}

None declared.

\section{REFERENCES}

1. Ljungberg B, Cowan NC, Hanbury DC, Hora M, Kuczyk MA, Merseburger AS, et al.: EAU guidelines on renal cell carcinoma: the 2010 update. Eur Urol. 2010; 58: 398-406.

2. Novick AC: Renal hypothermia: in vivo and ex vivo. Urol Clin North Am. 1983; 10: 637-44.

3. Thompson RH, Lane BR, Lohse CM, Leibovich BC, Fergany $A$, Frank I, et al.: Every minute counts when the renal hilum is clamped during partial nephrectomy. Eur Urol. 2010; 58: 340-5.
4. Nguyen MM, Gill IS: Halving ischemia time during laparoscopic partial nephrectomy. J Urol. 2008; 179: 627-32; discussion 632.

5. Baumert H, Ballaro A, Shah N, Mansouri D, Zafar N, Molinié V,et al.: Reducing warm ischaemia time during laparoscopic partial nephrectomy: a prospective comparison of two renal closure techniques. Eur Urol. 2007; 52: 1164-9.

6. Guillonneau B, Bermúdez H, Gholami S, El Fettouh H, Gupta R, Adorno Rosa J, et al.: Laparoscopic partial nephrectomy for renal tumor: single center experience comparing clamping and no clamping techniques of the renal vasculature. $J$ Urol. 2003; 169: 483-6.

7. Nadu A, Kitrey N, Mor Y, Golomb J, Ramon J: Laparoscopic partial nephrectomy: is it advantageous and safe to clamp the renal artery? Urology. 2005; 66: 279-82.

8. Gill IS, Eisenberg MS, Aron M, Berger A, Ukimura 0, Patil $\mathrm{MB}$, et al.: "Zero ischemia" partial nephrectomy: novel laparoscopic and robotic technique. Eur Urol. 2011; 59: 128-34

9. Ficarra V, Novara G, Secco S, Macchi V, Porzionato A, De Caro $R$, et al.: Preoperative aspects and dimensions used for an anatomical (PADUA) classification of renal tumours in patients who are candidates for nephron-sparing surgery. Eur Urol. 2009; 56: 786-93.

10. Porpiglia F, Volpe A, Billia M, Renard J, Scarpa RM: Assessment of risk factors for complications of laparoscopic partial nephrectomy. Eur Urol. 2008; 53:590-6.

11. Nuttall M, van der Meulen J, Emberton M: Charlson scores based on ICD-10 administrative data were valid in assessing comorbidity in patients undergoing urological cancer surgery. J Clin Epidemiol. 2006; 59: 265-73.

12. Kutikov A, Uzzo RG: The R.E.N.A.L. nephrometry score: a comprehensive standardized system for quantitating renal tumor size, location and depth. J Urol. 2009; 182: 844-53.

13. Dindo D, Demartines N, Clavien PA: Classification of surgical complications: a new proposal with evaluation in a cohort of 6336 patients and results of a survey. Ann Surg. 2004; 240: 205-13.

14. Go AS, Chertow GM, Fan D, McCulloch CE, Hsu CY: Chronic kidney disease and the risks of death, cardiovascular events, and hospitalization. N Engl J Med. 2004 23; 351: 1296-305. Erratum in: N Engl J Med. 2008;18: 4.

15. Touijer K, Guillonneau B: Advances in laparoscopic partial nephrectomy. Curr Opin Urol. 2004;14: 235-7.

16. Porpiglia F, Fiori C, Bertolo R, Angusti T, Piccoli GB, Podio $V$, et al.: The effects of warm ischaemia time on renal function after laparoscopic partial nephrectomy in patients with normal contralateral kidney. World J Urol. 2012; 30: 257-63.

17. Patil MB, Gill IS: Zero-ischaemia robotic and laparoscopic partial nephrectomy (PN). BJU Int. 2011; 108: 780-92.

18. $\mathrm{Ng} \mathrm{CK}$, Gill IS, Patil MB, Hung AJ, Berger AK, de Castro Abreu AL, et al.: Anatomic renal artery branch microdissection to facilitate zero-ischemia partial nephrectomy. Eur Urol. 2012; 61: 67-74. 
19. Cunningham AJ: Controlled hypotension to minimize blood loss of anaemic Jehovah's witness patient undergoing total hip and shoulder replacement. Br J Anaesth. 1982; 54: 895-8.

20. Ahlering TE, Henderson JB, Skinner DG: Controlled hypotensive anesthesia to reduce blood loss in radical cystectomy for bladder cancer. J Urol. 1983; 129: 953-4.

21. O'Connor PJ, Hanson J, Finucane BT: Induced hypotension with epidural/general anesthesia reduces transfusion in radical prostate surgery. Can J Anaesth. 2006; 53: 873-80.
22. Dutton RP: Controlled hypotension for spinal surgery. Eur Spine J. 2004; 13(Suppl 1): S66-71.

23. Rais-Bahrami S, George AK, Herati AS, Srinivasan AK, Richstone L, Kavoussi LR: Off-clamp versus complete hilar control laparoscopic partial nephrectomy: comparison by clinical stage. BJU Int. 2012; 109: 1376-81.

Correspondence address:

Dr. Francesco Porpiglia Division of Urology

Department of Clinical and Biological Sciences, University of Turin "San Luigi” Hospital Regione Gonzole 10 10043 Orbassano (Turin), Italy Fax: + 39011 903-8654 E-mail: porpiglia@libero.it 